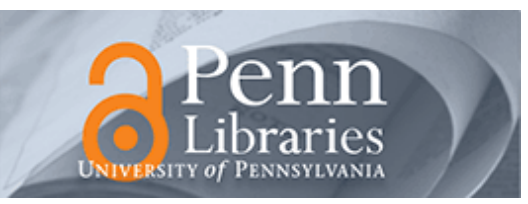

University of Pennsylvania ScholarlyCommons

November 2004

\title{
Rationalization of the plastic flow behavior of Polysynthetically- twinned (PST) TiAl crystals based on slip mode observation using AFM and Schmid's law
}

Yali Chen

University of Pennsylvania

David P. Pope

University of Pennsylvania, pope@seas.upenn.edu

Follow this and additional works at: https://repository.upenn.edu/mse_papers

\section{Recommended Citation}

Chen, Y., \& Pope, D. P. (2004). Rationalization of the plastic flow behavior of Polysynthetically-twinned (PST) TiAl crystals based on slip mode observation using AFM and Schmid's law. Retrieved from https://repository.upenn.edu/mse_papers/94

Copyright Materials Research Society. Reprinted from MRS Research Society Symposium Proceedings Volume 842.

2004 Fall Meeting Symposium

Integrative and Interdisciplinary Aspects of Intermetallics

Publisher URL:http://www.mrs.org/s_mrs/sec_subscribe.asp?CID=2702\&DID=115623\&action=detail

This paper is posted at ScholarlyCommons. https://repository.upenn.edu/mse_papers/94

For more information, please contact repository@pobox.upenn.edu. 


\title{
Rationalization of the plastic flow behavior of Polysynthetically-twinned (PST) TiAl crystals based on slip mode observation using AFM and Schmid's law
}

\author{
Abstract \\ PST TiAl samples of different orientations were prepared and deformed by compression at room \\ temperature. The deformation structures on the free surfaces were scanned using an AFM. It was found \\ that when the angle between the lamellar interfaces and the loading axis is between 20 degree and 80 \\ degree, PST samples deform primarily by shear in slip planes parallel to the lamellar interfaces. When the \\ angle is below 20 degree, both the gamma phase and the alpha 2 phase deform by shear in slip planes \\ inclined with the lamellar interfaces, but the shear vectors lie in the interface. When the angle is close to \\ 90 degree, complex deformation behavior occurs. Shear in planes parallel to the lamellar interfaces \\ contributes more to the overall strain in the directions perpendicular to the loading axis and the out-of- \\ plane shear contributes to the strain in the compression direction. The characteristic U-shape curve of the \\ yield stress versus the angle between the loading axis and the lamellar interfaces can be explained quite \\ well using different C.R.S.S. for the three different deformation modes.
}

\section{Comments}

Copyright Materials Research Society. Reprinted from MRS Research Society Symposium Proceedings Volume 842.

2004 Fall Meeting Symposium

Integrative and Interdisciplinary Aspects of Intermetallics

Publisher URL:http://www.mrs.org/s_mrs/sec_subscribe.asp?CID=2702\&DID=115623\&action=detail 


\title{
Rationalization of the plastic flow behavior of Polysynthetically-twinned (PST) TiAl crystals based on slip mode observation using AFM and Schmid's law
}

\author{
Yali Chen and David P. Pope \\ Department of Materials Science and Engineering, University of Pennsylvania \\ Philadelphia, PA 19104-6272, U.S.A
}

\begin{abstract}
PST TiAl samples of different orientations were prepared and deformed by compression at room temperature. The deformation structures on the free surfaces were scanned using an AFM. It was found that when the angle between the lamellar interfaces and the loading axis is between 20 degree and 80 degree, PST samples deform primarily by shear in slip planes parallel to the lamellar interfaces. When the angle is below 20 degree, both the gamma phase and the alpha 2 phase deform by shear in slip planes inclined with the lamellar interfaces, but the shear vectors lie in the interface. When the angle is close to 90 degree, complex deformation behavior occurs. Shear in planes parallel to the lamellar interfaces contributes more to the overall strain in the directions perpendicular to the loading axis and the out-of-plane shear contributes to the strain in the compression direction. The characteristic U-shape curve of the yield stress versus the angle between the loading axis and the lamellar interfaces can be explained quite well using different C.R.S.S. for the three different deformation modes.
\end{abstract}

\section{Introduction}

It is well known that the deformation behavior of polysynthetically twinned (PST) TiAl crystals changes with their orientations. When the loading axis is parallel or perpendicular with the lamellar interface, the yield stress is quite high. While when the loading axis is inclined with the lamellar interfaces, the crystal is relatively easy to be deformed. [1-4] This property was demonstrated by Kim with an irregular U-shaped yield stress-orientation curve. [3] Clearly, the change of the curve is not only related to the change of the applied shear stress on the deformation systems. In fact, TEM results $[1,4]$ have shown that at different orientation, different deformation systems are activated owing to the confinement of the lamellar interfaces. However, due to the limits of the TEM observation, it is hard to get a comprehensive picture of the plastic deformation behavior.

In our previous studies, the deformation behaviors at $\mathrm{A}, \mathrm{B}$ and $\mathrm{N}$ orientations were investigated using AFM. [5-7]The results can be summarized as a simple cartoon shown in figure 1. When the compression axis is parallel to the lamellar interface, i.e., in the so called A orientation, the gamma phase deforms primarily by shear in slip planes inclined with the lamellar interface.[5] In each gamma domain, the shear vectors lie in the lamellar interfacial plane, or the combination of shear vectors produce a zero strain across the lamellar interfaces in a scale of tens of nanometers. The alpha 2 phase deforms by prismatic slip, with shear vector also parallel to the lamellar boundaries. As a result, the microscopic and macroscopic strain in the direction perpendicular to the lamellar interfaces is zero when the PST crystals are in the A orientation.

When the compression axis is inclined by the lamellar interfaces by 45 degree (B orientation), the gamma phase deforms primarily by shear in slip planes parallel to the lamellar interfaces. [6] Shear in planes inclined with the lamellar interfaces also happens at high strain 
level, but contribute very little to the overall strain. The alpha 2 phase does not deform at this orientation because of the high C.R.S.S for the basal slip system. [8] The alpha 2 layers act as rigid plates and move along with the shear of the gamma lamellae.

When the loading axis is perpendicular to the lamellar interfaces, the deformation behavior is much more complicated. Shear parallel to the lamellar interfaces was found to be the primary deformation process at the low strain level, although the applied shear stress for this deformation system is zero. With the increase of strain level, cross lamellar shear becomes dominant. Prismatic slip in the alpha 2 phase was also observed at high strain level. These abnormal deformation behaviors were attributed to the coherency stress and stress concentration at the lamellar interfaces. [7]

For convenience, the three deformation modes are named $\mathrm{A}, \mathrm{B}$ and $\mathrm{N}$ deformation modes respectively, corresponding to the orientations of the PST samples. It can be seen from figure 1 that because of the selective activation of the deformation systems, the deformation of A and B oriented samples is quite anisotropic, as discovered by Kishida et al., [3,4] which might cause large strain incompatibility among lamellar grains in full-lamellar TiAl polycrystals if the grains are in these orientations. However, in the polycrystals, the lamellar grains are randomly oriented, so it is worthwhile to study the deformation behavior of PST crystals at some intermediate orientations. In this paper, the results from AFM observations will be presented and discussed.
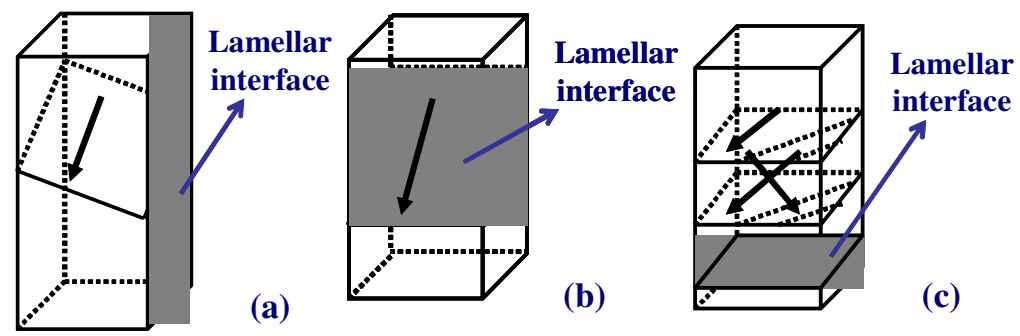

Figure 1. Schematic of the three deformation modes: A type (a), B type (b), $N$ type (c) (The bold arrows indicate the microscopic shear direction in each layer)

\section{Experimental details}

PST crystals with a nominal composition of Ti-48at\% Al were grown using optical floating zone furnace. Samples with different orientations were cut by Electrical Discharge Machining. The angle between the loading axis and the lamellar interfaces, $\varphi$, was set to be $10^{\circ}$, $20^{\circ}, 30^{\circ}, 70^{\circ}$ and $80^{\circ}$ as shown in figure 2 .

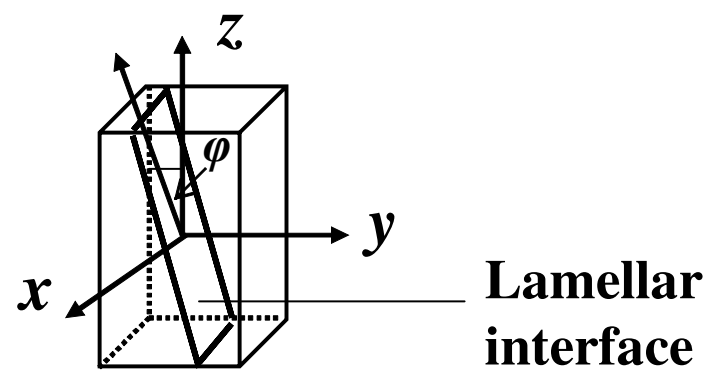

Figure 2 The geometry of PST samples. ( $\varphi$ is the angle between the compression axis and the lamellar interfaces.) 
The free surfaces of the samples were mechanically polished and electro-polished. Compression tests were carried out under room temperature at a speed of $1 \times 10^{-6} / \mathrm{s}$ using an Instron machine. The deformation structures emerged on the free surfaces were studied using a DI 3000 Dimension AFM. For detail discussion about the formation of deformation bands on the free surfaces and the convolution of the tip with the bands, please refer to the references 5-7.

\section{Results}

1. $\varphi=10^{\circ}$

Figure 3(a) is a deflection image showing the typical deformation structure when the loading axis inclines with the lamellar interfaces by 10 degree. The scan direction is parallel to the loading axis. It can be seen that the structure is similar to that of the A-oriented samples. [6] The lamellar interfaces are straight in the areas scanned. Some of the uphill deformation bands go across all the lamellae inside the scan area. The quantity of the downhill bands seems to be larger than that in the A-oriented samples. This might be because that the two slip planes which are symmetric in the A orientation are no longer symmetric. However, because of the requirements of strain compatibility and the condition of the local stress state, still only one type of band can freely go through the lamellar boundaries.

As with the A orientation, two types of gamma domains deform by super dislocation slip with Burger vectors parallel to the lamellar interfaces. The other four domains deform either by ordinary dislocation slip with Burger vector parallel to the lamellar interfaces, or by combination of ordinary dislocation slip and twinning with shear vectors inclined to the lamellar interfaces. The net result is still that there is no strain in the direction perpendicular to the lamellar boundaries.

The $\alpha_{2}$ lamellae can also deform freely in samples of this orientation as shown in figure 3(b). At this orientation, the Schmid factor for the prismatic slip is slightly lower than that for the A orientation, changing from 0.433 to 0.420 , so the deformation of the $\alpha_{2}$ lamellae is not strongly affected.
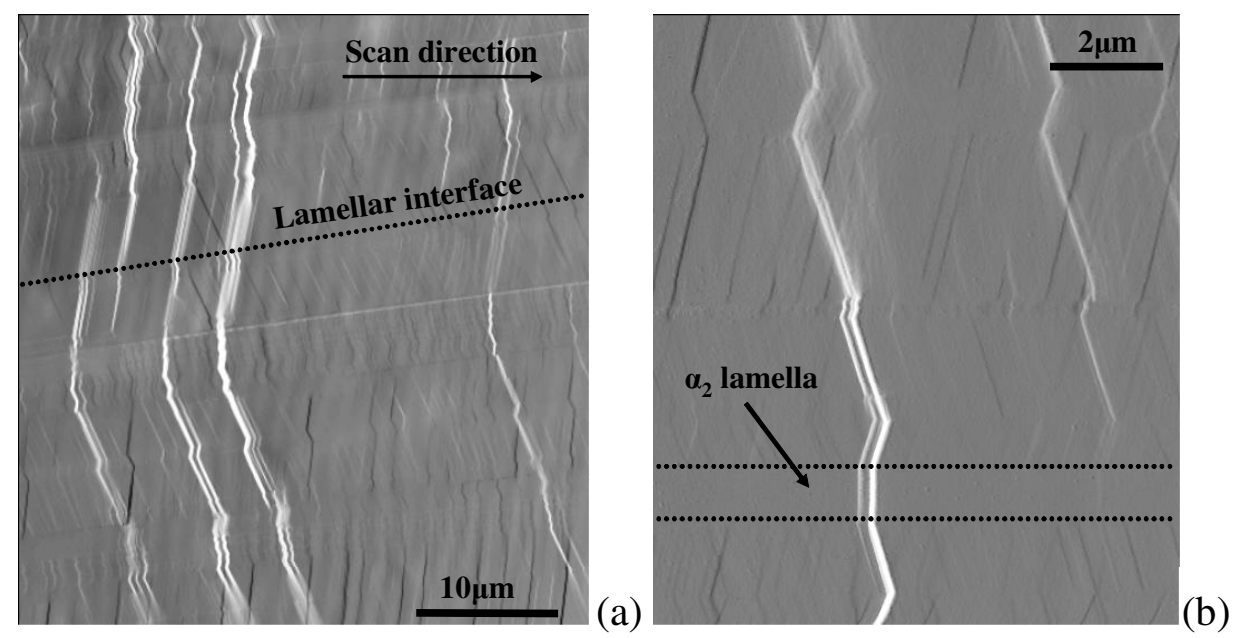

Figure 3 (a) The deformation structure on the $y z$ face of a sample deformed by $5 \%$ when $\varphi=10^{\circ}$. (Deflection image with scan direction parallel to the loading axis);

(b) The deformation structure in the $\alpha_{2}$ lamellae. (Deflection image with scan direction parallel to the lamellar interfaces) 


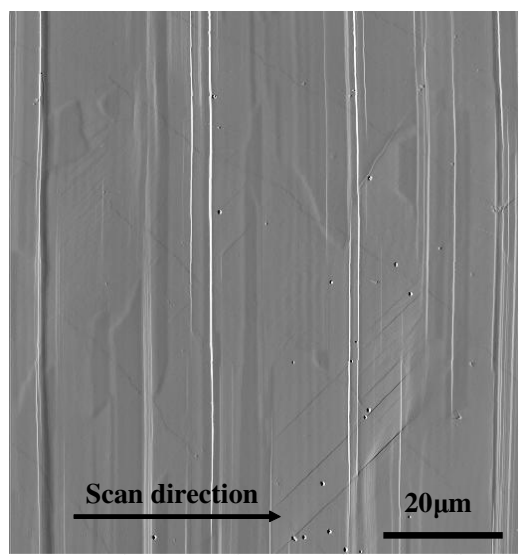

(a)

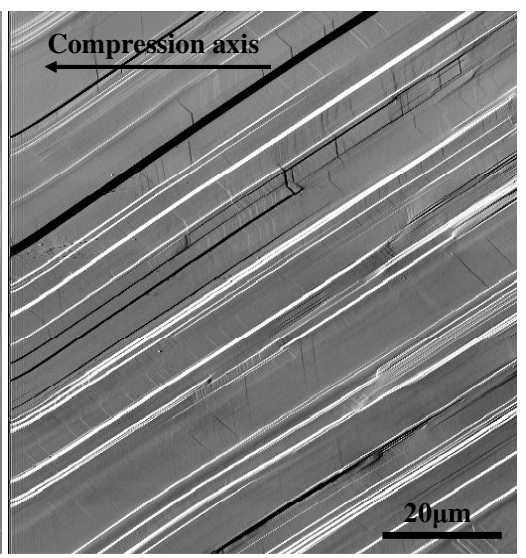

(b)

Figure 4 The deformation structure of a $\varphi=30^{\circ}$ sample deformed by $3.5 \%$ (a) $x z$ face; (b) $y z$ face. (Deflection images)

\section{2. $\varphi=20^{\circ}$ and $\mathbf{3 0}^{\circ}$}

Figure 4 shows the deformation structure of a sample deformed by $3 \%$ when the loading axis is inclined to the lamellar interfaces by $30^{\circ}$. Figure 4(a) is taken on the $x z$ face and figure 4(b) is taken on the $y z$ face. This deformation structure is similar to that of the B-oriented sample deformed by the same amount. [5] On the front $x z$ surface, only uphill parallel bands emerge. On the $y z$ side surface, both uphill and downhill bands appear which were formed by deformation systems with shear vectors having projections in the opposite directions. As with the B oriented samples, the macroscopic strain in the $x$ direction is zero. The samples with $\varphi=20^{\circ}$ have similar deformation structure.

The difference from the $\mathrm{B}$ orientation is that, at these orientations, there are more cross lamellar bands. This is reasonable since the resolved shear stresses on the slip planes other than (111) are larger than in the B orientation, especially for those deformation systems with shear vectors parallel to the lamellar interfaces. This feature is similar to type A orientation when the scan direction is parallel to the lamellar interfaces. However, these cross-lamellar bands contribute much less to the overall strain than the parallel bands. The height of the parallel bands varies from tens of nanometers to over 500 nanometers, while the step height of the cross lamellar bands is typically below $10 \mathrm{~nm}$, with only a very few having height of tens of nanometers.

Vertical bands perpendicular to the lamellar interfaces were not found in the areas scanned. The Schmid factor for the prismatic deformation systems is 0.318 for $\varphi=30^{\circ}$, about $75 \%$ of that for the A oriented samples. Also, because the yield stress at this orientation is much lower that that of the A oriented sample, the resolved shear stress might not be high enough for the prismatic slip systems to move. The basal slip systems cannot be activated either, since the Schmid factor is only 0.22 , smaller than that for the prismatic slip systems, while their C.R.S.S. is higher.

\section{2. $\varphi=70^{\circ}$ and $80^{\circ}$}

When the loading axis is inclined with the lamellar interfaces by $80^{\circ}$, the deformation structures are still similar to that of the B-oriented samples as shown in figure 5. On $x z$ face, only uphill parallel bands appear and on the $y z$ face, both uphill and downhill bands appear. The deformation structure of samples with $\varphi=70^{\circ}$ is similar to that shown in these figures. 


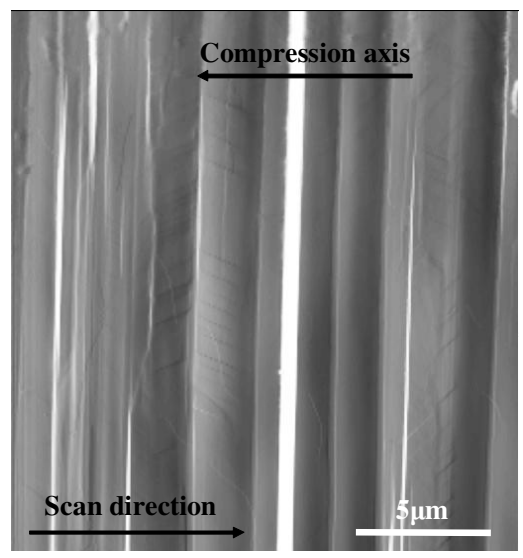

(a)

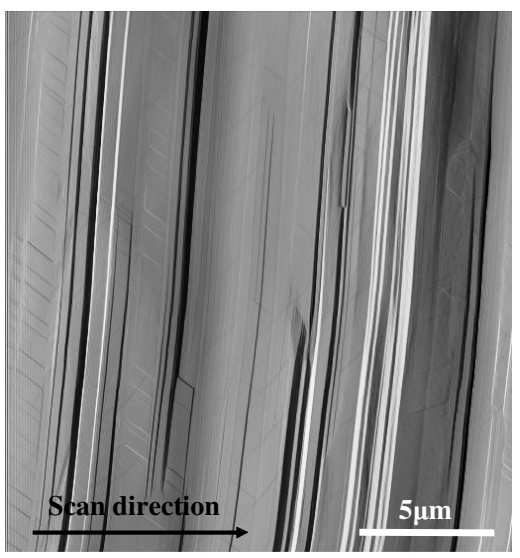

(b)

Figure 5 The deformation structure of a sample deformed by $2.8 \%$ when $\varphi=80^{\circ}$ (a) $x z$ face; (b) $y z$ face (Deflection images)

Cross lamellar bands also exist, especially for $\varphi=80^{\circ}$, but parallel bands still contribute more to the overall strain. As those in the $\mathrm{N}$ oriented, [7] the bands usually reside inside only one or several lamellae and do not extend for long distances. The lamellar interfaces no longer stay straight because these bands produce offsets of tens of nanometers perpendicular to the lamellar boundaries.

\section{Discussions and conclusions}

From the AFM results, it can be seen that the deformation behavior of the PST crystals at any orientation can be described by one or two of the three deformation modes discussed in the introduction part. When the angle between the loading axis and the lamellar interfaces is below 20 degrees, PST crystals deform mainly by the A deformation mode. When the angle is between 20 and 80 degrees, the B deformation mode is the primary one, with in-plane shear dominates. When the orientation is more close to A and $\mathrm{N}$ orientations, cross lamellar shear also occurs, but contribute much less to the overall strain. Only at orientations where the loading axis is very close to perpendicular to the lamellar interface, cross lamellar shear becomes dominate.

Although some theoretical calculations and experimental results have shown that twinning and ordinary dislocation slip might be the easiest deformation modes in the two-phase TiAl materials, $[1,4,9]$ it is still not clear which deformation mode controls the yield point of the PST specimens. According to the AFM results discussed above, there should be three C.R.S.S. values for the three deformation modes separately. Table 1 lists the calculations of the C.R.S.S of the deformation systems for different deformation modes, assuming that these deformation systems are the first ones to be activated and determines the yield stress. The values of the yield stresses are taken from Kim's results [3] for A, B, and $\mathrm{N}$ orientations.

Table 1 C.R.S.S in different deformation modes

\begin{tabular}{|c|c|c|c|c|}
\hline \multicolumn{2}{|c|}{ Deformation mode } & A & B & $\mathrm{N}$ \\
\hline \multicolumn{2}{|c|}{ Yield stress (MPa) ${ }^{[64]}$} & 690 & 200 & 800 \\
\hline \multirow{3}{*}{$\begin{array}{c}\text { C.R.S.S } \\
\text { (MPa) }\end{array}$} & Twinning & 162.84 & 86.6 & 125.6 \\
\hline & Ordinary dislocation & 281.52 & 100 & 217.6 \\
\hline & Superdislocation & 281.52 & 100 & 217.6 \\
\hline
\end{tabular}




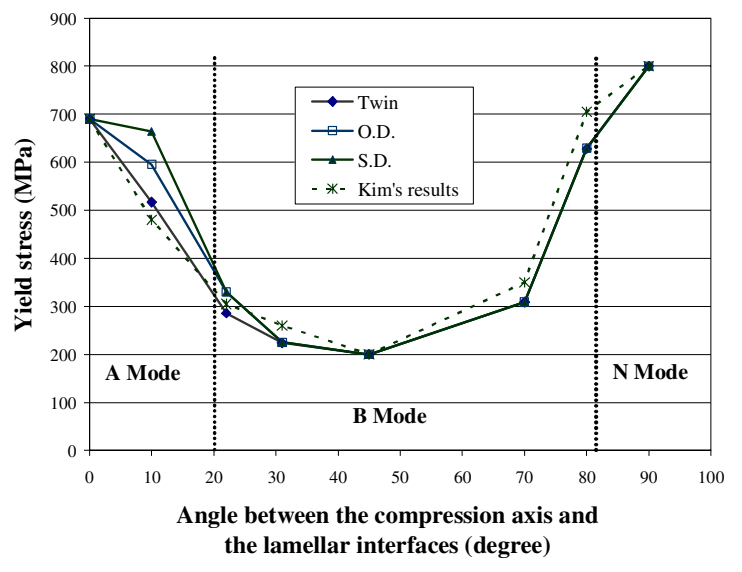

Figure 6 Comparison of the yield stress calculated based on different C.R.S.S

Using the C.R.S.S values from table 1 to calculate the yield stresses of the other orientations, based on the Schmid factors, gives the results shown in figure 6, accompanied by the results from Kim's result. The points with the angle below $20^{\circ}$ are calculated using A-type deformation C.R.S.S and those between $20^{\circ}$ and $80^{\circ}$ are calculated based on the B-type deformation C.R.S.S. The results calculated based on the twinning system is closest to the experimental results, especially in the A type deformation regime. So, it might be reasonable to conclude that twinning controls the onset of plastic deformation in PST crystals. The big change of yield stress from the A and $\mathrm{N}$ type to $\mathrm{B}$ type deformation mode is due to the difference of C.R.S.S. of twinning on slip planes parallel and those inclined to the lamellar interfaces.

The transition from one mode to another comes from the competition of the resolved shear stresses on different slip planes. When the resolved shear stress is above the C.R.S.S on the planes parallel to the lamellar interfaces, the B type mode will definitely take over because the confinement of the lamellar interfaces is avoided. Since most lamellar grains in the full-lamellar polycrystals should deform by A and B type deformation modes, which lead to anisotropic deformation, strain incompatibility will inevitably be produced at the grain boundaries. As a result, the deformability of the two-phase TiAl crystals with full-lamellar structure is limited.

\section{References}

1. H. Inui, A. Nakamura, M. H. Oh and M. Yamaguchi, Acta. Metall. Mater., 40 (11), 3095 (1992)

2. Y. Umakoshi, T. Nakano, Acta Metall. Mater., 41, 1155 (1993 )

3. M. Kim, M. Nomura, V. Vitek and D. Pope, in in High-Temperature-Ordered Intermetallic Alloys VIII, edited by E. George, M. Mills, M. Yamaguchi, (Mater. Res. Soc. Symp. Proc. 552, Boston, MA, 1998) pp KK3.1.1

4. K. Kishida, H. Inui, M. Yamaguchi, Philos. Mag. A, 78 (1) 1 (1998)

5. Y. Chen, D. Pope, Microscopy Research and Techniques, submitted

6. Y. Chen, D. Pope, AFM study of the plastic deformation of Polysynthetically twinned (PST) TiAl crystals in hard orientations — I: A orientation, Acta Mater., to be submitted

7. Y. Chen, D. Pope, AFM study of the plastic deformation of Polysynthetically twinned (PST) TiAl crystals in hard orientations — II: N orientation, Acta Mater., to be submitted

8. H. Inui, M. H. Oh, A. Nakamura, M. Yamaguchi, Philos. Mag. A, 66 (4), 539 (1992)

9. Lu L, Pope DP, Mater. Sci. and Eng. A, 239-240, 126 (1997) 